\title{
Binocular contrast interactions in two-frame motion discrimination
}

\author{
A. Raghunandan \\ Michigan College of Optometry, Ferris State University, Big Rapids, Michigan \\ AND
}

S. B. STEVENSON

University of Houston, College of Optometry, Houston, Texas

\begin{abstract}
Previous studies have shown that two-frame motion detection thresholds are elevated if one frame's contrast is raised, despite the increase in average contrast - the "contrast paradox." In this study, we investigated if such contrast interactions occurred at a monocular or binocular site of visual processing. Two-frame motion direction discrimination thresholds were measured for motion frames that were presented binocularly, dichoptically or interocularly. Thresholds for each presentation condition were measured for motion frames that comprised either matched or unmatched contrasts. The results showed that contrast mechanisms producing the contrast paradox combine contrast signals from both eyes prior to motion computation. Furthermore, the results are consistent with the existence of monocular and binocular contrast gain control mechanisms that coexist either as combined or independent systems.
\end{abstract}

It has been shown previously that two-frame motion discrimination thresholds are elevated if one frame's contrast is raised, despite the increase in average contrast (Stevenson \& Cormack, 2000). This effect is paradoxical as any simple combination or correlation model (Adelson \& Bergen, 1985; van Santen \& Sperling, 1985; Watson \& Ahumada, 1985) predicts an overall increase in the signalto-noise ratio and hence should predict an improvement in discrimination - a "contrast paradox." Such an effect is not exclusive to motion but has also been shown to also occur in Vernier (Stevenson \& Cormack, 2000) and stereoscopic mechanisms (Cormack, Stevenson, \& Landers, 1997; Halpern \& Blake, 1988; Legge \& Gu, 1989; Schor \& Heckmann, 1989; Stevenson \& Cormack, 2000).

In the case of stereopsis, it has been postulated that the mechanisms producing the "paradox" effect may not be exclusively monocular (Halpern \& Blake, 1988). The observations of Schor and Heckmann (1989) and Kontsevich and Tyler (1994) provided further evidence for the existence of strong binocular/interocular contrast masking mechanisms between eyes that may account for the paradox effect observed with stereopsis. However, in the case of motion processing, previous studies have postulated that motion mechanisms are essentially monocular, because presentation of interocular motion sequences lacked the spatiotemporal (Lu \& Sperling, 2001), Fourier sensitive characteristics (Georgeson \& Shackleton, 1989) and phasespecific contrast facilitation (Georgeson, 1988) displayed by monocular motion sequences. It therefore follows that if the contrast paradox observed with two-frame motion reflects inherent contrast interactions prior to or within motion mechanisms, then such contrast interactions must exert their impact monocularly - that is, independent of the contrast interactions in the contralateral eye. Evidence for the existence of such contrast mechanisms linked to motion processes have been provided by a number of previous studies (Adelson \& Bergen, 1985; Georgeson \& Scott-Samuel, 1999; Lu \& Sperling, 1996; Morgan \& Chubb,1999; Rainville, Scott-Samuel, \& Makous, 2002; Stone \& Thompson, 1992; Stromeyer, 2003). However, none of these studies have provided explicit evidence regarding the level at which such putative mechanisms may reside.

The aim of the present study was to use the contrast paradox observed in two-frame motion as a probe to infer the level at which such contrast interactions affect motion processing. The present study entailed binocular, dichoptic and interocular contrast manipulations of the motion frames comprising a two-frame motion sequence. With binocular presentation both eyes viewed the same contrast sequence of motion frames (Experiment 1, Figure 1A). In the case of dichoptic presentation (Experiment 2, Figure 1B), each eye viewed the opposite contrast sequence of motion frames. For example, if the sequence of motion frames presented to right eye was the high contrast frame followed by the low contrast frame, then the sequence of motion frames presented to the left eye was the low contrast frame followed by the high contrast frame. In the interocular condition (Experiment 3, Figure 1C); motion

A. Raghunandan, raghuna@ferris.edu 


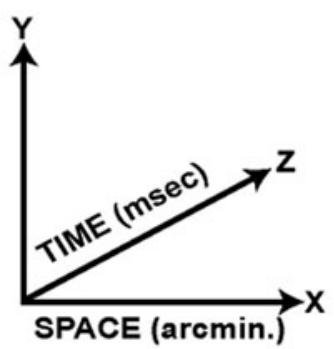

\section{A. Experiment 1 \\ Both left and right eyes viewed the same contrast sequence.}

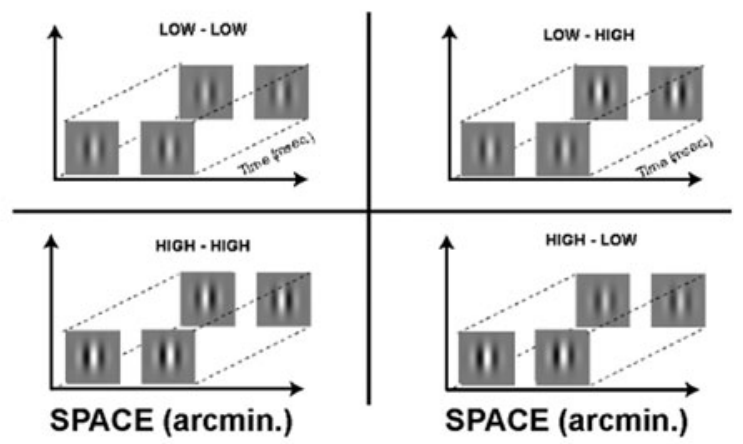

\section{B. Experiment 2 Matched contrast sequences were the same as experiment 1. Mixed contrast sequences were unmatched across eyes and across time.}

\section{Experiment 3 Motion frames were presented interocularly with the non-stimulated eye viewing mean luminance.}
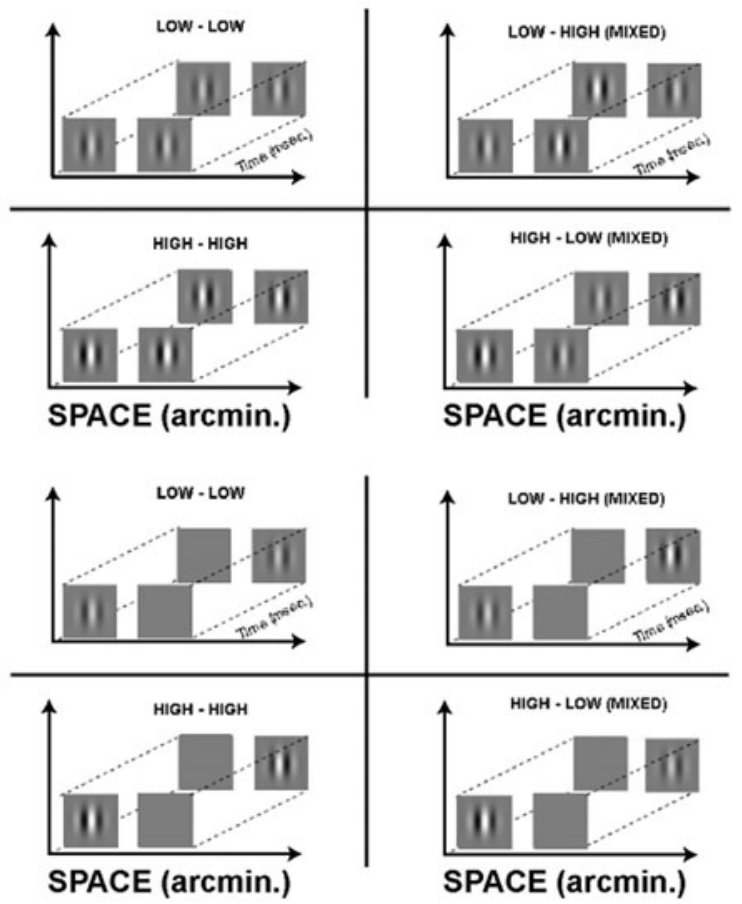

Figure 1. Each quadrant in each subplot depicts the space-time plot for each of the four contrast sequences used in Experiments 1 (A), 2 (B), and 3 (C). The $x$-, $y$-, and $z$-axes are as indicated in the top left figure. Each pair of gratings represents the gratings presented to the left and right eyes.

frames were presented across eyes. For example, if the first frame was presented to the right eye, the left eye viewed mean luminance, and this was then followed by the second frame being presented to the left eye while the right eye viewed mean luminance. The results are consistent with the existence of binocular contrast gain control mechanisms, but leaves open the possibility for the existence of monocular contrast gain control mechanisms that may coexist with binocular mechanisms either as combined or independent systems.

\section{METHOD}

\section{Observers}

Three subjects (two authors and a graduate student from the research group) served as observers. All subjects were well-trained psychophysical observers.

\section{Stimuli}

Stimuli were vertical Gabor patches presented as a two-frame movie on a linearized Apple eMac CRT monitor using the Psychophysics Toolbox (Brainard, 1997; Pelli, 1997). Each frame of the motion sequence was presented for $133 \mathrm{msec}(10$-video frames at a frame rate of approximately $13.33 \mathrm{msec}$ per frame) in immediate succession. The carrier frequency was $1 \mathrm{cpd}$ and the space constant of the Gaussian was 43 arcmin. Motion was produced through phase shifts of the carrier. Stimuli were viewed dichoptically through a mirror haploscope. At the $149-\mathrm{cm}$ viewing distance (this includes the $12-\mathrm{cm}$ distance added by the mirrors of the haploscope), the angular subtense of the stimulus was $4.26^{\circ}$ with each pixel subtending 1 arc minute. Mean luminance as measured by a Minolta LS110 Photometer was $56 \mathrm{~cd} / \mathrm{m}^{2}$ at the viewing distance.

\section{Procedure}

Prior to the start of each block of trials, subjects nulled their associated phoria by adjusting the mirrors of the haploscope to achieve alignment of Nonius lines presented in two blank square regions 
$\left(4.26^{\circ} \times 4.26^{\circ}\right)$, having the same spatial dimensions and mean luminance as the test stimuli. A keypress initiated the presentation of the test stimulus after which a blank stimulus of luminance equal to the mean luminance of the test stimuli was presented. Subjects indicated the perceived direction of motion by a keypress that also initiated the next trial. All stimuli were viewed against a dark surround. Each Gabor had a contrast of either 0.1 or 0.4 , producing two matched contrast motion sequences (low to low and high to high) and two mixed contrast sequences (low to high and high to low). The contrast sequences mentioned above refer to the progression of the contrast with time. For example, "high to low" refers to conditions where the high contrast frame was presented first followed by the low contrast frame. Table 1 presents the contrast sequences of the motion frames for each of the three Experiments.

A single block comprised 25 repetitions of nine phase shifts in each contrast condition, randomly interleaved in a single interval, forced choice, method of constant stimuli. Thresholds were calculated as the standard deviation of the best-fitting cumulative normal to the psychometric functions of the 4 contrast conditions.

\section{RESULTS}

\section{Experiment 1}

In Experiment 1 (Figure 1A) both eyes saw the same sequence (low to high, high to low, high to high, or low to low).

The results for Experiment 1 are plotted in Figure 2. Minimum motion thresholds are plotted on the vertical axis against Gabor contrast on log-log scales. Thresholds for the mixed contrast conditions are plotted against an abscissa value of 0.2 . This value corresponds to the geometric mean contrast of the motion frames $(0.1$ and $0.4)$. Motion discrimination thresholds improved slightly with increasing contrast $(0.1$ to 0.4$)$ for the matched contrast conditions. Motion thresholds for unmatched contrast conditions (low to high and high to low) were significantly elevated, and high-to-low thresholds were consistently higher than low-to-high thresholds across all subjects. These results are consistent with previously published reports (Stevenson et al., 2000) and therefore established the validity of the present stimulus paradigm in producing the "contrast paradox" observed with twoframe motion. Increasing overall contrast improves performance, but increasing one component's contrast degrades performance.

\section{Experiment 2}

In Experiment 2, the stimuli were the same as Experiment 1 , but each eye saw the opposite mixed-contrast sequence. If the left eye saw a low-to-high sequence (low contrast frame followed by high contrast frame), the right eye saw a high-to-low sequence (high contrast frame followed by the low contrast frame), and vice versa (see Figure 1B). Thus contrast frames are mixed dichoptically. The matched contrast conditions were the same as in Experiment 1.

The logic of Experiment 2 was as follows. In the mixed contrast sequences, each eye was presented with a contrast sequence that produced a "contrast paradox" in Experiment 1 (e.g., low to high in the left eye and high to low in the right eye). Even though there was a change in contrast within an eye from Frame 1 to Frame 2, the average contrast across eyes remained constant. If the contrast mechanisms implicated in the contrast paradox affected motion processing exclusively at a monocular level, then motion direction discrimination thresholds for the mixed contrast sequences will be elevated. However, if the contrast mechanism affected motion processing at a binocular stage, then motion direction discrimination thresholds should be equivalent to mean contrast performance - that is, the absence of a contrast paradox effect would imply that contrast mechanisms affect motion processing after some form of binocular combination.

The results for Experiment 2 are plotted in Figure 3. The $x$ - and $y$-axes are the same as in Experiment 1 . The data clearly reveal an absence of the contrast paradox effect as observed in Experiment 1. Mixed contrast thresholds are equivalent to the average performance for matched low and matched high contrasts, consistent with the inference that the contrast gain control mechanisms operating in motion direction discrimination must occur at or beyond a site of binocular combination.

\section{Experiment 3}

The result of Experiment 2 is suggestive of the operation of a motion system located at a binocular site. We therefore investigated whether motion frames presented interocularly exhibited similar contrast interactions. In Experiment 3, the first motion frame was presented to one

Table 1

Contrast of Each Frame of the Motion Sequence As a Function of Experimental Condition

\begin{tabular}{|c|c|c|c|c|c|c|c|c|c|}
\hline \multirow[b]{2}{*}{ Experiment } & \multirow[b]{2}{*}{ Frame } & \multicolumn{2}{|c|}{ Matched High } & \multicolumn{2}{|c|}{ Mixed Low to High } & \multicolumn{2}{|c|}{ Mixed High to Low } & \multicolumn{2}{|c|}{ Matched Low } \\
\hline & & Left Eye & Right Eye & Left Eye & Right Eye & Left Eye & Right Eye & Left Eye & Right Eye \\
\hline 1 & 1 & 0.4 & 0.4 & 0.1 & 0.1 & 0.4 & 0.4 & 0.1 & 0.1 \\
\hline 2 & 1 & 0.4 & 0.4 & 0.1 & 0.4 & 0.4 & 0.1 & 0.1 & 0.1 \\
\hline & 2 & 0.4 & 0.4 & 0.4 & 0.1 & 0.1 & 0.4 & 0.1 & 0.1 \\
\hline 3 (Rightward & 1 & 0.4 & 0.0 & 0.1 & 0.0 & 0.4 & 0.0 & 0.1 & 0.0 \\
\hline interocular) & 2 & 0.4 & 0.0 & 0.4 & 0.0 & 0.1 & 0.0 & 0.1 & 0.0 \\
\hline
\end{tabular}

Note-Frame 1 and Frame 2 refer to the first and second frames of the motion sequence, respectively. Data for the mixed high-tolow and mixed low-to-high conditions in Experiment 2 were averaged. Similarly, the data for the rightward and leftward interocular sequences for Experiment 3 were averaged and presented as the interocular condition in Figure 4. 

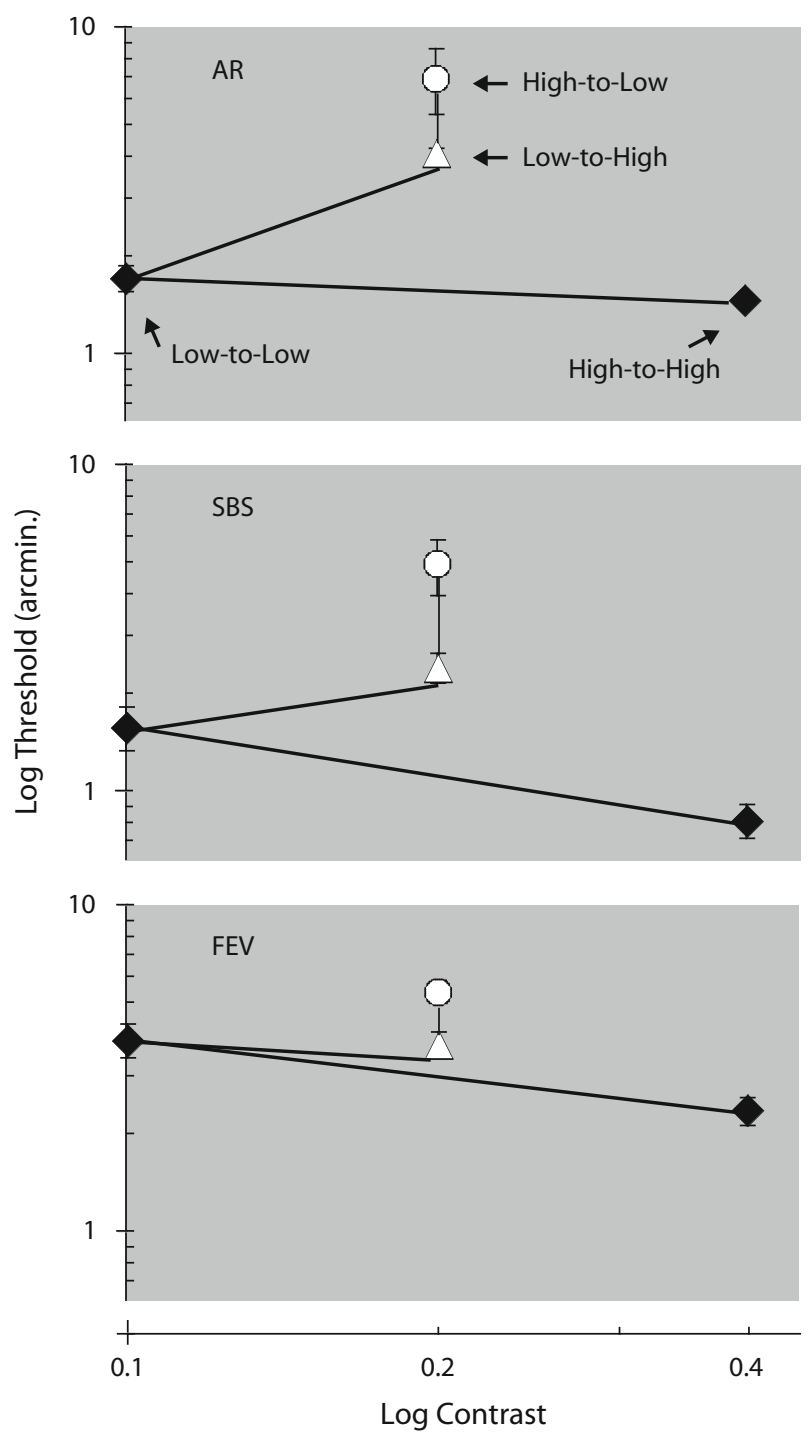

Figure 2. Two-frame motion thresholds (mean $\pm 1 S E M$ ) for the four contrast conditions (matched low, matched high, low-to-high, and high-to-low contrast sequences) are plotted for 3 subjects. The $x$-axis represents the contrast of the frames plotted on log scale. Mismatched contrast sequences are plotted against a geometric mean contrast of 0.2 (triangles, low to high; circles, high to low). Note that mismatched contrast sequences produced thresholds much higher than predicted by geometric mean contrast performance-contrast paradox. Furthermore, thresholds were higher if the high contrast frame preceded the low contrast frame.

eye followed by the presentation of the second frame to the other eye. The nonstimulated eye viewed a background of mean luminance during the interocular condition. The interocular contrast sequence was either presented with the matched sequence (low to low and high to high) or the mixed sequences (low to high and high to low (see Figure 1C).

Minimum interocular motion thresholds were significantly elevated for both matched and mixed interocular contrast sequences (Figure 4). Minimum interocular motion thresholds for the low-to-high sequence were consistent with mean contrast performance when referenced to the matched interocular contrast sequence - that is, no contrast paradox. However, high-to-low thresholds were significantly elevated such that thresholds could not be measured with the range of disparities used (as indicated by the dashed vertical line extending from the low-high interocular threshold). This does not necessarily mean that high-to-low thresholds are immeasurable but rather thresholds were larger than the 180-degree phase displacement limit (30 arcmin for our 1-cpd gratings).

\section{DISCUSSION}

The results of the present study have confirmed the generality of the paradox effect when extended to twoframe motion processing. Presenting motion frames with unequal contrasts adversely affects motion perception when compared to conditions where the motion frames have matched contrasts. A simple Reichardt type system, without contrast normalization of some kind, predicts that motion energy will vary proportionally to the product of the contrasts of the two frames comprising the two-frame motion sequence (van Santen \& Sperling, 1984). It therefore follows that increasing the contrast of one or both frames of the two-frame motion sequence will increase motion energy and hence improve performance. It is for this reason that the elevation of two-frame motion thresholds with mismatched contrasts has been referred to as paradoxical. However, it has been shown that the effect of mismatched contrast frames on motion detection or discrimination is dependent on the overall magnitude of the contrast of the frames comprising the motion sequence (Georgeson, 1988; Morgan \& Chubb, 1999; Solomon et al., 2005). For very low contrast frames close to detection thresholds, increasing the contrast of one frame improves motion performance (Georgeson, 1988; Morgan \& Chubb, 1999; Morgan \& Cleary, 1992; Solomon, Chubb, John, \& Morgan, 2005; van Santen \& Sperling, 1984), however at moderate contrast levels (approximately two to three times the contrast threshold for direction discrimination of matched contrast sequences), mismatched contrast frames adversely affects motion processing (Georgeson, 1988; Morgan \& Chubb, 1999; Stevenson \& Cormack, 2000; Stromeyer, 2003). The transition from facilitation to inhibition with increasing contrast has been attributed to the recruitment of contrast gain control mechanisms that operate in conjunction with motion processing (Morgan \& Chubb, 1999; Stevenson \& Cormack, 2000). The contrasts employed in the present study were sufficiently high to invoke such gain control mechanisms; therefore, it is not surprising that motion thresholds increased as the contrast of one frame of a two-frame motion sequence was increased. Previous reports have explored the effects of pedestal contrasts and the spatiotemporal effects of surround contrast on motion gain control mechanisms ( $\mathrm{Lu}$ \& Sperling, 1996; Rainville et al., 2002), however in this study we show that motion gain control mechanisms are also influenced by the contrast of the frames comprising the motion sequence itself.

There are two pertinent observations in the present results that provide additional clues to the site(s) at which 

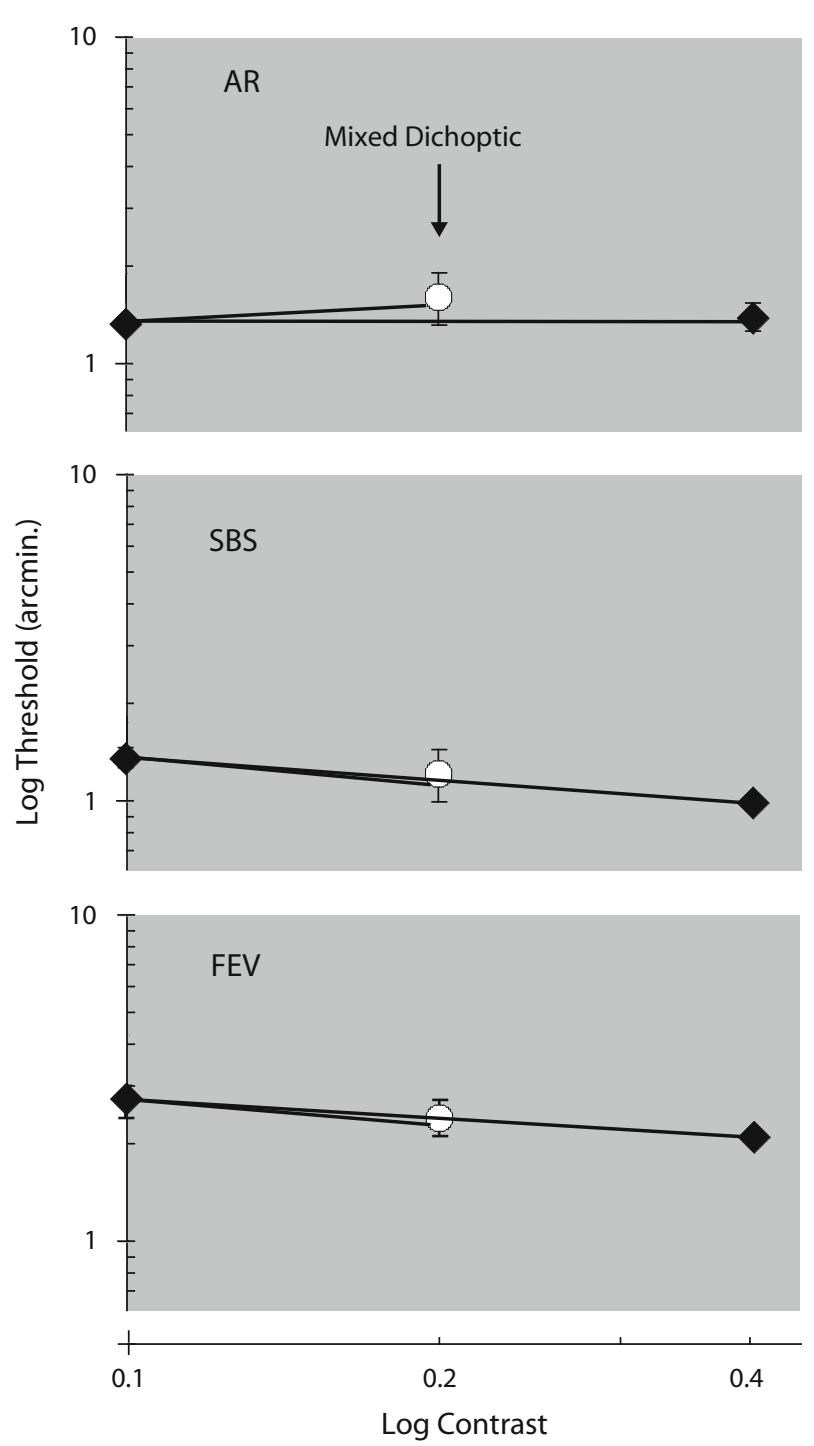

Figure 3. Results for Experiment 2: Matched contrast conditions are plotted as diamonds and the mean of the dichoptic "mixed" sequence is plotted as circles (mean $\pm 1 S E M)$. When motion frames are presented with mixed contrast frames across eyes and across time (dichoptic mixed sequences), motion thresholds were comparable to geometric mean contrast performance-no contrast paradox.

such gain control processes may occur. The first observation is that the magnitude of the paradox effect was dependent on the temporal sequence of the motion frames when their contrasts were mismatched. Minimum motion thresholds were affected greater if the high contrast frame preceded the low contrast frame (high-to-low conditions). This temporal asymmetry implies that some contrast-gain control occurs prior to the multiplicative or correlative stages in a Reichardt type motion detector and specifically predicts that the contrast of the first motion frame is weighted much higher in setting the gain for the subsequent motion frame. Conversely, if the gain control system occurred at or after the multiplicative stage of a Reichardt detector, such temporal asymmetry would fail to occur due to the commutability property of the multiplicative stage (Morgan \& Chubb, 1999). Stromeyer (2003) and Morgan and Chubb (1999) reported similar observations.

The second pertinent observation was that when motion frames with mismatched contrast sequences were presented dichoptically (Experiment 2), measured performance was equivalent to geometric mean contrast, even though each eye received a contrast sequence that was effective in producing a paradox. If motion computation were confined to monocular systems, then performance for the dichoptic condition (Experiment 2) should be equivalent to the performance predicted by the mismatched contrast sequences presented to each eye (Experiment 1). However, this was not the case. Therefore, it follows that contrast signals from each eye must combine prior to motion computation in order to produce a central contrast sequence that is matched. This result places motion computation mediating threshold for the dichoptic condition (Experiment 2) at a post binocular site, but leaves open the possibility of less sensitive monocular motion mechanisms. What does this mean for gain control? We performed an informal simple iteration with a divisive normalization scheme and found that some form of a centrally located binocular gain control mechanism is required after summation of monocular signals and prior to or in conjunction with motion computation to capture the trend depicted by the present results. Considering both Experiments 1 and 2 together, then, our results indicate that both monocular and binocular contrast interactions occur prior to motion extraction. Postulations of separable monocular and binocular mechanisms that contribute to motion processing have been also revealed by studies investigating the motion adaptation aftereffect (van Kruysbergen \& de Weert, 1993, 1994). These studies (van Kruysbergen \& de Weert, 1993, 1994) posited the existence of independent monocular systems and two types of binocular systems, namely, AND-gated (activated by input from both eyes) and OR-gated (activated by inputs from either eye) type mechanisms. Therefore, it is not unreasonable to assume that while the results of Experiment 2 may reflect the output of the postulated AND-gated system, it is equally likely that the results of Experiment 1 may reflect the output of the monocular and/or the OR-gated type systems noted above.

In addition to the above, electrophysiological recordings from primate and nonprimate cortical cells (Hubel, 1995) have shown quite definitively, the existence of such separable monocularly and binocularly driven cells. With specific regard to motion gain control systems, electrophysiological results from cat cortical simple cells showed no correlation between ocular dominance and the presence of gain control (Truchard, Ohzawa, \& Freeman, 2000), suggesting that both monocular and binocular driven cortical simple cells comprise contrast gain control systems. Furthermore, the same study (Truchard et al., 2000) postulated the existence of binocular simple cells comprising both monocular and centrally located binocular contrast gain control mechanisms involved in motion processing. These findings seem consistent with the psychophysical results obtained in the present study. We therefore speculate that the present psychophysical results are strongly 


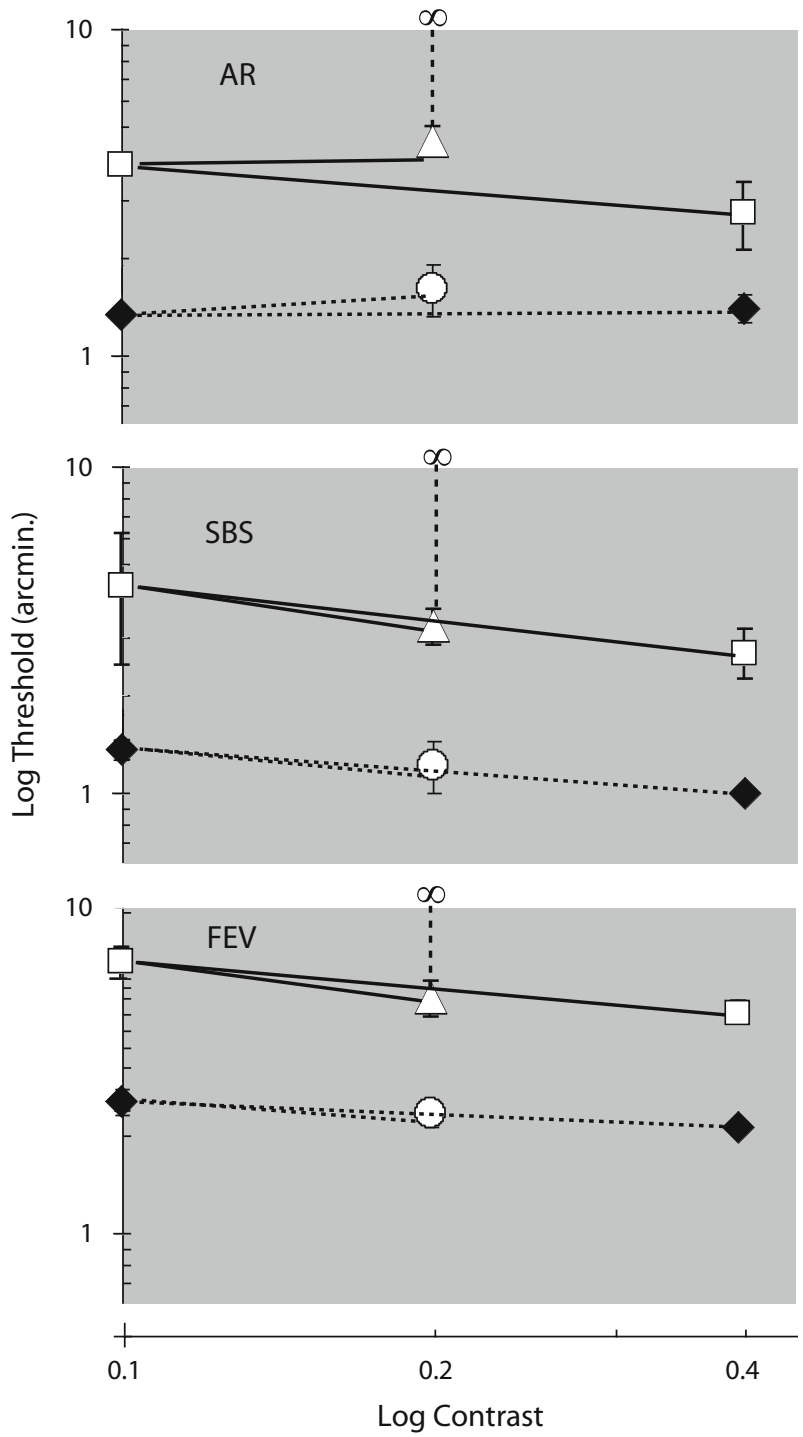

Figure 4. Results for Experiment 3 (interocular motion): Matched contrast conditions are plotted as squares and mismatched sequences as triangles. Each datum represents the mean motion threshold $( \pm 1 S E M)$ for both leftward and rightward interocular motion sequences. Thresholds for the high-tolow contrast sequences exceeded the 180-degree phase shift limit and therefore could not be measured in the present paradigm (represented as a dashed line extending to infinity, $\infty$ ). The results from Experiment 2 (diamonds and circles connected by fine dashed lines) are plotted for comparison. Motion thresholds for matched interocular contrast sequences are approximately fourfold higher compared with the results of Experiment 2 regardless of contrast sequence.

suggestive of central binocular gain control systems that operate in conjunction with motion processing in normal human visual systems and that they may coexist either as systems combined with or independent of monocular contrast gain control systems.

The results of Experiment 2 imply the existence of motion detectors that combine information from both eyes prior to motion computation. In Experiment 2 each eye received a contrast sequence that was effective in produc- ing the paradox effect, however when presented dichoptically, performance was better than the performance predicted by the contrast sequence presented to either eye. This observation is at odds with reports that propose that motion computation by dedicated motion energy detectors (first-order systems) is confined to monocular systems (Georgeson, 1988; Georgeson \& Shackleton, 1989; Lu \& Sperling, 2001). Shadlen and Carney (1986) and later Carney and Shadlen $(1992,1993)$ reported that flickering gratings presented with interocular spatiotemporal quadrature phase display characteristics comparable to monocular first-order motion systems. They therefore posited the existence of interocular first-order motion mechanisms that combined information from both eyes prior to motion computation. The results of Experiment 2 are consistent with the assertions of Carney and Shadlen (1992, 1993) and Carney (1997). To this effect we measured two-frame motion thresholds when motion frames are presented interocularly. Both Lu and Sperling (2001) and Carney and Shadlen (1992) have suggested that this nonoptimal method of stimulus presentation taps into feature tracking or third order motion systems. The results of Experiment 3 show clearly that both matched and unmatched interocular contrast sequences produced thresholds that were significantly higher than the dichoptic condition (Experiment 2). Therefore, the dichoptic condition produced motion thresholds that are comparable to motion thresholds predicted by first order motion systems and is significantly better than the predicted performance of interocular third order or feature tracking motion systems.

In summary, the present study provides evidence for the existence of binocular gain control systems that operate in conjunction with motion processing. It is possible that gain control mechanisms residing at monocular levels may also coexist with binocular gain control systems. Furthermore, the results from the dichoptic condition allude to the existence of motion detectors that combine information from both eyes prior to motion computation. Threshold performance in this condition was more consistent with first-order motion systems than interocular third order or feature tracking motion systems.

\section{AUTHOR NOTE}

This research was supported in part by NIH Grant R01-EY12986 awarded to S.B.S. Correspondence concerning this article should be addressed to A. Raghunandan, Michigan College of Optometry, Ferris State University, 1310 Cramer Circle, Big Rapids, MI 49307 (e-mail: raghuna@ferris.edu).

\section{REFERENCES}

Adelson, E. H., \& Bergen, J. R. (1985). Spatiotemporal energy models for the perception of motion. Journal of the Optical Society of America A, 2, 284-299.

Brainard, D. H. (1997). The Psychophysics Toolbox. Spatial Vision, 10, 433-436.

CARNEY, T. (1997). Evidence for an early motion system which integrates information from the two eyes. Vision Research, 37, 2361-2368.

Carney, T., \& Shadlen, M. N. (1992). Binocularity of early motion mechanisms: Comments on Georgeson and Shackleton. Vision Research, 32, 187-191.

Carney, T., \& Shadlen, M. N. (1993). Dichoptic activation of the early motion system. Vision Research, 33, 1977-1995. 
Cormack, L. K., Stevenson, S. B., \& Landers, D. D. (1997). Interactions of spatial frequency and unequal monocular contrasts in stereopsis. Perception, 26, 1121-1135.

Georgeson, M. A. (1988). Spatial phase dependence and the role of motion detection in monocular and dichoptic forward masking. Vision Research, 28, 1193-1205.

Georgeson, M. A., \& Scott-Samuel, N. E. (1999). Motion contrast: A new metric for direction discrimination. Vision Research, 39, 43934402.

Georgeson, M. A., \& Shackleton, T. M. (1989). Monocular motion sensing, binocular motion perception. Vision Research, 29, 1511-1523.

HALPERN, D. L., \& BLAKE, R. R. (1988). How contrast affects stereoacuity. Perception, 17, 483-495.

Hubel, D. H. (1995). Eye, brain, and vision. New York: Scientific American Library.

Kontsevich, L. L., \& Tyler, C. W. (1994). Analysis of stereothresholds for stimuli below $2.5 \mathrm{c} / \mathrm{deg}$. Vision Research, 34, 2317-2329.

LegGe, G. E., \& Gu, Y. (1989). Stereopsis and contrast. Vision Research, 29, 989-1004.

Lu, Z. L., \& Sperling, G. (1996). Contrast gain control in first- and second-order motion perception. Journal of the Optical Society of America A, 13, 2305-2318.

Lu, Z. L., \& SPERLING, G. (2001). Three-systems theory of human visual motion perception: Review and update. Journal of the Optical Society of America A, 18, 2331-2370.

Morgan, M. J., \& ChubB, C. (1999). Contrast facilitation in motion detection: Evidence for a Reichardt detector in human vision. Vision Research, 39, 4217-4231.

Morgan, M. J., \& Cleary, R. F. (1992). The effect of contrast substitutions upon motion detection in spatially random patterns. Vision Research, 32, 639-643.

Pelli, D. G. (1997). The VideoToolbox software for visual psychophysics: Transforming numbers into movies. Spatial Vision, 10, 437-442.

Rainville, S. J. M., Scott-Samuel, N. E., \& Makous, W. L. (2002). The spatial properties of opponent-motion normalization. Vision Research, 42, 1727-1738
Schor, C., \& Heckmann, T. (1989). Interocular differences in contrast and spatial frequency: Effects on stereopsis and fusion. Vision Research, 29, 837-847.

Shadlen, M., \& CARney, T. (1986). Mechanisms of human motion perception revealed by a new cyclopean illusion. Science, 232, 95-97.

Solomon, J. A., Chubb, C., John, A., Morgan, M. (2005). Stimulus contrast and the Reichardt detector. Vision Research, 45, 2109-2117.

Stevenson, S. B., \& Cormack, L. K. (2000). A contrast paradox in stereopsis, motion detection, and vernier acuity. Vision Research, $\mathbf{4 0}$, 2881-2884.

Stone, L. S., \& Thompson, P. (1992). Human speed perception is contrast dependent. Vision Research, 32, 1535-1549.

Stromeyer, C. F., III (2003). Temporal asymmetry in motion masking: A shortening of the temporal impulse response function. Vision Research, 43, 261-268.

Truchard, A. M., Ohzawa, I., \& Freeman, R. D. (2000). Contrast gain control in the visual cortex: Monocular versus binocular mechanisms Journal of Neuroscience, 20, 3017-3032.

van Kruysbergen, N. A. W. H., \& de Weert, C. M. M. (1993). Apparent motion perception: The contribution of the binocular and monocular systems. An improved test based on motion aftereffects. Perception, 22, 771-784.

van Kruysbergen, N. A. W. H., \& de Weert, C. M. M. (1994). Aftereffects of apparent motion: The existence of an AND-type binocular system in human vision. Perception, 23, 1069-1083.

van Santen, J. P. H., \& Sperling, G. (1984). Temporal covariance model of human motion perception. Journal of the Optical Society of America A, 1, 451-473.

VAn Santen, J. P. H., \& Sperling, G. (1985). Elaborated Reichardt detectors. Journal of the Optical Society of America A, 2, 300-321.

Watson, A. B., \& Ahumada, A. J. (1985). Model of human visualmotion sensing. Journal of the Optical Society of America A, 2, $322-$ 341

(Manuscript received August 2, 2006; revision accepted for publication February 13, 2007.) 